Dokumentation

Rahel Beyer

\title{
Tagung „Leichte Sprache - verständliche Sprache“ am Institut für Deutsche Sprache (Mannheim) vom 19.-20. Oktober 2017
}

In den letzten Jahren haben Anstrengungen in Politik und Gesellschaft zur Einbeziehung aller Menschen ins öffentliche Leben deutlich zugenommen. Ein Teilthema dieser Inklusionsbemühungen stellt die „Leichte Sprache“ dar. Dabei handelt es sich um einen Ansatz, barrierefreie Kommunikation zu ermöglichen, indem Texte für sprachlich eingeschränkte Menschen verständlich gestaltet werden. Daneben gibt es schon länger verwandte Phänomene wie etwa „einfache Sprache“ und „bürgernahe Sprache“ mit ähnlichem Adressatenkreis.

Um dem wachsenden Interesse an diesem Thema Rechnung zu tragen, veranstaltete das Institut für Deutsche Sprache (IDS) gemeinsam mit der Gesellschaft für deutsche Sprache (GfdS) in Verbindung mit dem Deutschen Sprachrat vom 19. bis 20. Oktober 2017 ein Kolloquium mit dem Titel „Leichte Sprache - Verständliche Sprache“. Im Rahmen des Kolloquiums sollten der Phänomenbereich „Sprachvereinfachung“, sein Hintergrund und die empirische Überprüfung diskutiert werden. Ziel dieser Veranstaltung war es, die akademische Perspektive mit derjenigen der in der Praxis mit Leichter Sprache befassten Akteure zusammenzubringen. Entsprechend befanden sich unter den eingeladenen Referenten Vertreter aus verschiedenen Bereichen.

Das Kolloquium gliederte sich in zwei Teile: Für den Abend des 19. Oktober war zunächst eine Podiumsdiskussion angesetzt, an die sich am Freitagvormittag eine Reihe von Fachvorträgen anschloss.

Nach der Eröffnung des Kolloquiums bzw. des Abends durch Ludwig M. Eichinger, Direktor des Instituts für Deutsche Sprache, stellte der Moderator Albrecht Plewnia (IDS) zunächst die teilnehmenden Diskutanten vor und gab ihnen die Gelegenheit zu einem kurzen Statement, in dem sie ihre Position und ihr Interesse am Thema darlegten.

Gisela Holtz (Netzwerk „Leichte Sprache“, Münster) skizzierte hierbei die historische Entwicklung des Netzwerks „Leichte Sprache“. Seine Gründung geht zurück auf das Jahr 2006, in dem sich einige Büros und Akteure, die sich mit Leichter Sprache beschäftigten, zusammengetan haben, um sich untereinander abzustimmen und gleiche Texte mit gleich hoher Qualität zu gestalten. Außerdem entschloss man sich dazu, Prüfgruppen, bestehend aus Menschen mit Lernschwierigkeiten (so die Selbstbezeichnung), zu konsultieren, so dass auch mit den Betroffenen und nicht (nur) über sie gesprochen wird. In der Praxis stellt sich jedoch oft heraus, dass es nicht einfach ist, mit linguistischen Laien Texte zu diskutieren. Über die Jahre hat sich dieses Netzwerk inzwischen deutlich vergrößert: 2013 zählte das Netzwerk ca. 120 Mitglieder, darunter Privatpersonen, Übersetzungsbüros und soziale Einrichtungen wie die AWO, die Lebenshilfe u.a. Ein Anliegen des Netzwerks für die Zukunft ist die Einrichtung eines Ausbildungsgangs für Leichte-Sprache-Übersetzer, 
um die Arbeit zu professionalisieren. Schließlich ist zu beobachten, dass viele Städte und Gemeinden gerne eigenständige Büros für Leichte Sprache einrichten würde, jedoch Geld und eine langfristig ausgerichtete Motivation fehlen.

Ursula Bredel (Universität Hildesheim) vertrat die sprachwissenschaftliche Perspektive; sie nannte drei Bereiche, in denen sprachwissenschaftliche/sprachdidaktische Erkenntnisse einer Weiterentwicklung der Leichte-Sprache-Praxis unterstützen könnten. Dazu gehört zum ersten die Verstehens- und Verständlichkeitsforschung: Das Wissen über das Verstehen und die Verständlichkeit von bestimmten Konstruktionen und Möglichkeiten und Grenzen ihrer Ersetzbarkeit liefert wichtige Anhaltspunkte für die Gestaltung von Leichte-Sprache-Texten. Zweitens ist an die Textlinguistik zu denken: Letztlich geht es nicht darum, einzelne Sätze regelkonform zu formulieren, sondern ganze Texte zu vermitteln. Entsprechend ist über Anforderungen an die Texte und an Textsorten - und deren Umsetzbarkeit angesichts eines reduzierten Sprachrepertoires - nachzudenken. Schließlich könnte die Sprachdiagnostik Hinweise bezüglich der Allgemeingültigkeit oder Zielgruppenspezifik von Sprach- und Leseeinschränkungen und den Folgen für die Texterstellungspraxis liefern. Noch nicht ganz klar ist die Eignung der Leichten Sprache als Instrument des Lernens (z.B. im Bereich DaZ).

Arne Janssen (GfdS, Berlin) berichtete aus der Arbeit des Redaktionsstabs am Deutschen Bundestag. Im Rahmen der allgemeinen Sprachberatung werden dort auch Aufträge im Bereich Leichte und v.a. Einfache Sprache realisiert. So wurde z.B. eine Rede eines Bundestagsabgeordneten in Einfache Sprache übertragen, Broschüren werden in Einfacher Sprache erstellt, und auch aus dem Petitionsausschuss werden Aufträge erteilt. Eine große Herausforderung stellt dabei die Vermittlung der Rechtssprache bzw. der Fachwörter dar. Zurzeit wird die Broschüre „Parlamentsdeutsch - Lexikon der parlamentarischen Begrif$\mathrm{fe}^{\text {" }}$ in Einfache Sprache übersetzt. Die konsultierten Prüfgruppen haben durchaus interessante Vorschläge gemacht (z.B. „Beschlussvorschlag“ statt „Beschlussempfehlung“). Adressaten von Einfacher Sprache werden dabei auf dem Alpha-Level 4 eingeordnet, Leichte-Sprache-Adressaten darunter. Bei allen Projekten gilt es, den Spagat zwischen juristischer Korrektheit, Verständlichkeit, den Wünschen der Auftraggeber und den Bedürfnissen der Adressaten zu schaffen.

Brigitte Vogel-Janotta (Deutsches Historisches Museum, Berlin) beschrieb ebenfalls ihre Erfahrungen in der Anwendung von Leichter Sprache im öffentlichen Bereich. Vor einigen Jahren haben sie und ihr Team aus der Abteilung „Bildung und Vermittlung“ angefangen, sich mit dem Thema Leichte Sprache zu beschäftigen und seitdem viel dazu gelernt, was sowohl die Platzierung als auch die Qualität der Texte betrifft. Andererseits stoßen sie noch immer auf einige Probleme: Diese reichen von einem erhöhten Platzbedarf der (Raum-)Texte in Leichter Sprache, über die Kompromissfindung zwischen Regelbefolgung, Wissensvermittlung (Funktionalität) und Prüfgruppenurteil sowie die Heterogenität der Adressaten bis hin über mangelnde Akzeptanz der Formulierungen von Seiten des Kuratoriums, der „Standardbesucher“ (Bildungsbürgertum) und der größeren Öffentlichkeit (Presse). Immer wieder sehen sie sich mit heftigen Vorwürfen einer Verfälschung von Tatsachen und einem herabgesenkten inhaltlichen wie sprachlichen Niveau konfrontiert. 
Kernpunkte der anschließenden Diskussion, die später auch für das Publikum geöffnet wurde, waren die Beschreibung von Verständlichkeit als Kontinuum, die Heterogenität der Adressaten (Menschen mit Lernschwierigkeiten, Funktionale Analphabeten, DemenzPatienten usw.), deren Bedürfnisse u.U. durch unterschiedliche Textsorten gestillt werden müssen, die bereits vorhandene Vielfalt von Begriffen und Ansätzen in der Sprachvereinfachung (Leichte Sprache, Einfache Sprache, klare Sprache usw.), die Zusammensetzung und Rolle der Prüfgruppen, die Subjektivität von Verstehen (vs. Objektivierung von Verständlichkeit mittels entsprechender Forschung) sowie nicht zuletzt die Position der Betroffenen und ihre eventuelle Enteignung bzw. Ausgrenzung gerade durch die Bereitstellung von gesondertem Material durch Nicht-Betroffene.

Die Reihe von Fachvorträgen wurde am Freitagmorgen von Ursula Bredel mit einem Vortrag zur „Leichten Sprache und Inklusion - am Beispiel der inklusiven Schulen“ eröffnet. Sie definierte Inklusion als „Normatives Gesellschaftsmodell, das auf der Idee einer maximal autonomen (individuelle Dimension) Partizipation (soziale Dimension) aller Gesellschaftsmitglieder fußt“. Bezogen auf Sprach-/Leseschwierigkeiten, die dieser Partizipation im Wege stehen, stellt die Leichte Sprache als reduzierte Varietät des Deutschen ein Instrument zur entsprechenden Aufbereitungen von Texten und zur Überwindung von Verstehensbarrieren dar. Bezugspunkt der erörterten Überlegungen waren Lernarrangements, in denen Kinder/Jugendliche mit und ohne sprachlichem Unterstützungsbedarf gemeinsam lernen. Hier kann ein Lösungsansatz für eine autonome Textrezeption für alle Schüler/innen darin bestehen, Paralleltexte in Leichter Sprache als intralinguale Übersetzungen anzubieten. Bredel nannte sechs Dilemmata, die im Zusammenhang mit Leichter Sprache auftreten (können): So enthält sie z.B. ein gewisses Diskriminierungspotenzial ihrer Nutzer/innen, das zusammen mit dem zugewiesenen Sonderstatus zu einer Etablierung und Verfestigung von Eigenkulturen (Abschottung) führen kann (Exklusionsdilemma). Zweitens kann es zu einem Teufelskreis von geringer Leistungserwartung, dem Gebrauch von Leichter Sprache und einem geringen Lerneffekt kommen (Attribuierungsdilemma). Des Weiteren ist es sehr schwierig, literarische Texte (bzw. Verfassertexte allgemein) adäquat in Leichte Sprache zu übersetzen, da sie dadurch zu expositorischen Texten werden (Textdilemma). Das vierte Dilemma liegt in der Verfehlung aller Eigenschaften der Bildungssprache (die per se ein Lernziel ist und sich durch ein spezifisches Inventar an lexikalischen, morphosyntaktischen und textlichen Mitteln auszeichnet), sowie der fachlichen Komplexität, die sich u.a. in Fachwörtern manifestiert (Bildungsdilemma). Fünftens ist in einer parallelen Anordnung die unterschiedlich(e) Länge der beiden Texte problematisch (Quantitätsdilemma). Schließlich können unterschiedlich (gestaltete) Texte einen kommunikativen Austausch zwischen den Schüler/innen erschweren, der für gemeinsames Lernen unabdingbar ist (Kommunikationsdilemma).

Im zweiten Vortrag stellte Bettina M. Bock (Universität zu Köln) „Empirische Untersuchungen zur Leichten Sprache“ vor, die im Rahmen des Projekts „Leichte Sprache im Arbeitsleben“ (LeiSA) durchgeführt wurden. Im linguistischen Teilprojekt wurden die Aspekte Wortschatz, Worttrennung, Grammatikverstehen (konkret am Beispiel des Genitivs), Textverstehen, Textsortenwissen, Makrotypografie und Text-Bild-Beziehungen fokussiert und eine Triangulation einer Vielzahl von Zugängen und Methoden (z.B. EyeTracking, mündliche Befragung, Lautes Denken/Erinnern) vorgenommen. Ausgangspunkt der Untersuchungen war die Annahme, dass die Zielgruppe(n) tendenziell unterschätzt werden sowohl was die Lesekompetenzen als auch das Wissen zu prototypischen 
grafischen Gestaltungen von Textsorten betrifft. Daten aus einer leitfadengestützten retrospektiven Befragung von zwei Personen mit der Zuweisung , geistige Behinderung ' bestätigten diese Annahme: Obwohl beide ein niedriges bzw. sehr niedriges Leseniveau (LeaLevel 3 bzw. 2) besitzen, erreichten sie ein adäquates Textverständnis eines Beispieltexts mit Brandschutzhinweisen. Auch eine Einverständniserklärung wurde trotz der niedrigen diagnostizierten Lesekompetenz auf der Basis von vorhandenen Wissensbeständen auch zu Textsorten gut verstanden. Als Fazit wurde als Alternative zur bisherigen starren Regelbasierung eine kontextbezogen angemessene Textgestaltung vorgeschlagen.

Ebenfalls eine empirische Überprüfung verschieden komplexer Sprachvarietäten nahmen Silvia Hansen-Schirra und Silke Gutermuth (Universität Mainz/Germersheim) vor. Dabei stellten sie außerdem verschiedene Probandengruppen gegenüber. So bekamen Studierende (Kontrollgruppe), Senioren, Menschen mit Migrationshintergrund sowie Menschen mit kognitiven Einschränkungen jeweils vier Texte (gleichen Inhalts) in Einfacher, in Leichter Sprache und im Original vorgelegt. Alle Probandengruppen (außer den Studierenden) beurteilten die Texte in Einfacher Sprache als verständlicher als die Originaltexte und die Texte in Leichter Sprache noch einmal verständlicher als die in Einfacher Sprache. Die Probanden mit Migrationshintergrund gaben für die Texte in Leichter Sprache das höchste Rating der Verständlichkeit ab. Der methodische Schwerpunkt der Studie lag auf psycholinguistischen Verfahren. So wurde mittels Eyetracking (Aufzeichnung der Blickbewegung) die Erstfixationsdauer (First Fixation Duration), die Fixationsdauer (Gaze Duration) sowie die Gesamtlesezeit gemessen. Auf der sprachlichen Ebene wurden Komposita fokussiert, die in den Leichte-Sprache-Texten in der Mediopunktvariante, d.h. mit einem Punkt zwischen den Kompositionsgliedern auf halber Höhe, geschrieben wurden. Im Ergebnis ließ sich festhalten, dass alle Probandengruppen vom Mediopunkt profitieren, und zwar sowohl hinsichtlich einer kürzeren Gesamtlesedauer der Komposita als auch bezüglich der ersten Fixation. Bei Letzterem war bei den Probanden mit Migrationshintergrund ein sehr starker Effekt zu beobachten. Texte in Leichter Sprache im Allgemeinen sowie Komposita im Speziellen scheinen also leichter verarbeitbar zu sein.

Mit dem Vortrag von Sibylle Hallik (GfdS, Berlin) zur „Leichten und Einfachen Sprache“ erweiterte sich der Fokus. Im ersten Teil diskutierte sie verschiedene Herangehensweisen, einen hochwertigen Text in Leichter Sprache zu produzieren. Dabei kann man sich z.B. an den Regeln des Netzwerks Leichte Sprache und/oder dem Ratgeber von Bredel/Maas (2015) orientieren, um ,regelkonforme Texte“ zu verfassen. Es gibt jedoch einige Unterschiede zwischen den beiden Werken. Ein weiterer Orientierungspunkt sind die Urteile der Prüfgruppen. Aber einige der mit einem Prüfsiegel versehenen Texte (beispielsweise des Netzwerks Leichte Sprache) weichen an manchen Stellen von den Regeln ab. Außerdem enthielt der Vortrag ein Plädoyer für ,gute“ Leichte Sprache/Einfache Sprache: Dies heißt u.a. Einhalten der Regeln der Orthografie, Festlegen bestimmter Formalien und vor allem Verzicht auf bestimmte Formen der Ellipse sowie die Verwendung ,sinnvoller“ Erklärungen. Im dritten Teil stellte Hallik das Projekt „Rentenpolitik in Einfacher Sprache“ vor. Es hat zum einem die schwierige Kompromissfindung zwischen (möglichst größer) Regelkonformität, den Lesekompetenzen der Prüfgruppe/Adressaten und den Vorstellungen des Auftraggebers gezeigt, zum anderen, dass auch Texte, die den Regeln der Einfachen Sprache entsprechen, für die Zielgruppe „Menschen mit Leseschwierigkeiten“ aufgrund der Komplexität des Inhaltes eine große Herausforderung darstellen können. 
Den abschließenden Vortrag zur „Fairständlichen Verwaltungssprache“ hielt Lutz Kuntzsch (GfdS, Wiesbaden). Er definierte Verwaltungssprache als „Gesamtheit der Kommunikation in (staatlichen) Einrichtungen, die eine verwaltende Funktion ausüben“ - sowohl nach außen mit den Rezipienten als auch intern zwischen den Angehörigen der jeweiligen Verwaltung. Zudem wies er ihr eine Brückenfunktion zwischen Rechtssprache als Fachsprache (Merkmal: Rechtsstaatlichkeit/Verwaltungshandeln) und der „Allgemeinsprache/bürgernahen Sprache“ (Merkmal: nicht fachlich gebunden, ,alltagsweltlich“) zu, die versucht, einen Kompromiss zwischen syntaktisch verdichteten und schriftsprachlichen Strukturen mit eindeutiger und erschöpfender Terminologie und offeneren Texten, die näher an der mündlichen Kommunikation sind und mehr erklärende Begriffe enthalten, zu schließen. In Seminaren der GfdS mit Mitarbeitern v.a. aus Stadtverwaltungen aus dem gesamten Bundesgebiet und Landesregierungen werden Grundsätze einer einfachen und bürgernahen Verwaltungssprache allgemein vermittelt. Zum Portfolio der GfdS gehören außerdem Textbearbeitung und Sprachberatung, die zusammen mit den Seminaren eine Sensibilisierung für die Notwendigkeit verständlicher sowie gleichzeitig juristisch abgesicherter Texte in der Verwaltungskommunikation erreichen möchte. Dabei handelt es sich um einen ständigen Aushandlungsprozess zwischen den beiden Zielen. Verständlichkeit umfasst die drei Ebenen Wortwahl (Bevorzugung von kurzen, gängigen Wörtern, Vermeidung von Substantivierungen usw.), Satzbau (z.B. Vermeidung der Verbalklammer) und Textaufbau (klare Gliederung, Einsatz vom Textgestaltungselementen usw.). Darüber hinaus soll über einen höflichen, freundlichen Ton, persönliche Ansprache und geschlechtergerechte Formulierungen eine gewisse Bürgernähe aufgebaut werden.

In seinem kurzen Abschlusswort der - angesichts kontroverser Diskussionen und eines großen Publikums erfolgreichen - Tagung bedankte sich Peter Schlobinski (Vorsitzender der GfdS, Hannover) bei allen Beteiligten und regte an, dass sich Wissenschaft und Praxis auch in der Zukunft weiter austauschen. 